\title{
Arbor
}

\section{Algunas reflexiones sobre Gaia, evolución y cultura}

\author{
Antonio Pou
}

Arbor CLXXII, 677 (Mayo), 127-134 pp.

Durante los viajes que James Lovelock realizó por todo el mundo, rastreando el DDT y otros componentes traza con su invento del detector de captura de electrones, le llamó la atención la interconectividad química de toda la atmósfera con los seres vivos y su gran estabilidad, lo que le llevó a la concepción de una nueva teoría, la hipótesis Gaia, que él define como "Una nueva visión de las interacciones entre las partes vivas $e$ inorgánicas del planeta. De ahi ha surgido la hipótesis, el modelo, en el cual la materia viva, el aire, los océanos y la superficie terrestre forman un sistema complejo que puede ser visto como un único organismo y que tiene la capacidad de mantener nuestro planeta como un lugar apto para la vida».

Lyn Margulis, la conocida bióloga, dotó a la hipótesis de una base científica que la dió credibilidad, pero en mi parecer el éxito de la teoría entre el gran público radica más en la elección del nombre «Gaia», la antigua madre-diosa griega, y su posible connotación religiosa, que no en su aportación científica. El mismo Lovelock da pie a esa interpretación cuando afirma «... para mí, Gaia es un concepto religioso y científico a la vez, y es manejable en ambas esferas», aunque en otros momentos ha intentado matizar «De ningún modo veo a Gaia como un ser consciente, un Dios alternativo. Para mí Gaia está viva y es parte del universo inefable y yo soy una parte de ella».

Desde el mundo científico se acepta mal esa ambivalencia y todavía hay quienes arquean las cejas al oir hablar de Gaia. Sin embargo la hipótesis ha facilitado un nuevo marco de referencia en el que ahora caben perspectivas más amplias e interesantes, como la simbiogénesis que propone Margulis. 
En el continuo debate sobre la evolución, y desde el punto de vista de su éxito social, es casi inevitable tratar de comparar Gaia con el evolucionismo de Darwin, aunque Lovelock se declara cien por cien darwinista y afirma que su hipótesis no lo reemplaza, sino que meramente lo extiende. Darwin suministró a la sociedad de su época argumentos que encajaban perfectamente con el discurso social al uso y que dieron justificación a las políticas de su nación. Lovelock ha proporcionado una nueva vía de pensamiento a la juventud inconformista de los años setenta de los países desarrollados, pero es dudoso que su impacto sea tan profundo y duradero como han sido las ideas darwinistas.

En un ambiente de globalización como el actual, en el que la información es un bien al alcance de muchos, parecería llegado el momento de tratar de acometer, de nuevo, una amplia reflexión sobre el tema de la evolución, sin pre-juicios, sin pre-concepciones, teniendo en cuenta la base conceptual que proporcionaría el análisis del conocimiento socio-científico-cultural que tenían las civilizaciones del pasado, junto con el que existe en el conjunto de las civilizaciones del mundo de hoy; sin confundir las ideas que del mundo se hace la civilización occidental con la realidad misma, o con la única posibilidad que tiene el ser humano de conocer. Para ello es imprescindible hacerse previamente una idea de la magnitud de lo que estamos discutiendo y de nuestras capacidades como seres, en este estadio evolutivo.

En el último cuarto del siglo XX ha visto la luz una nueva idea sobre la organización de la vida sobre la Tierra: la hipótesis Gaia. Curiosamente, esta nueva hipótesis no procede de alguien del campo de la biología o de las ciencias naturales, sino de un químico muy prestigioso: James Lovelock.

Pensador e inventor, diseñó y construyó en 1957 un detector de captura de electrones, con el que se pudo realizar por primera vez un seguimiento de las trazas del DDT por todo el mundo. Como fruto de sus recorridos analizando gases traza por muchos lugares del planeta, e impregnado por el hecho de que la interconectividad química de la atmósfera es una realidad obvia para cualquier químico o físico, extendió de forma natural sus observaciones a los intercambios gaseosos con los seres vivos. Sorprendido ante la estabilidad de los sistemas bióticos/abióticos, llegó a la formulación de una hipótesis que, según sus propias pala- 
bras, es «Una nueva visión de las interacciones entre las partes vivas e inorgánicas del planeta. De ahí ha surgido la hipótesis, el modelo, en el cual la materia viva, el aire, los océanos y la superficie terrestre forman un sistema complejo que puede ser visto como un único organismo y que tiene la capacidad de mantener nuestro planeta como un lugar apto para la vida».

Como puede verse, la idea básica es más bien un postulado que una hipótesis, puesto que parece de difícil demostración. Sin embargo es una idea que surgió en un momento histórico muy oportuno, en el que se comenzaba a reaccionar contra el racionalismo a ultranza de las décadas anteriores. El aire romántico de los setenta fue un caldo muy apropiado para la aceptación de la hipótesis Gaia, sobre todo entre los movimientos ambientalistas del momento. Por otra parte, el soporte entusiasta que la bióloga Lynn Margulis dió a la hipótesis, permitió dotarla de una base naturalista más firme y le dió credibilidad ante una parte importante de la sociedad científica. No obstante, en mi opinión, un componente esencial del éxito social de la hipótesis Gaia es el propio nombre, que fue magistralmente elegido por William Golding, un novelista amigo de Lovelock.

Gaia, Gaea, Gea, o GE, es el nombre que los antiguos griegos daban a la madre diosa, antes de que los helénicos introdujeran al dios masculino Zeus. Después pasó a encarnar a la diosa Tierra, y como tal es la que da los sueños, la que nutre a las plantas y a los niños pequeños. Aunque hoy la partícula geo (geografía, geología, geodinámica, etc) denota un carácter un tanto materialista, en la palabra «Gaia» recupera con su sonido una parte del aire místico que tuvo cuando era cantada por Homero. Muy probablemente sea precisamente ese componente seudo religioso de la hipótesis el que atrae a una buena parte de sus seguidores, que buscan en ella un sustituto algo más racional a sus inquietudes religiosas, en un mundo moderno en el que las ideas tradicionales han quedado quizá un tanto desfasadas. Releyendo a Lovelock, da la impresión que él mismo partió de esa situación; de hecho afirma: «...para mí, Gaia es un concepto religioso y científico a la vez, y es manejable en ambas esferas.»

$\mathrm{Al}$ situarse en esa posición, Lovelock incurre en una cierta incorrección social dentro de la comunidad científica, donde no está bien visto el mezclar temas aparentemente tan separados. En «Las Edades de Gaia»1 su posición se hace totalmente explícita al hacer una interpretación de la figura de la Virgen María en relación con Gaia; interpretación que probablemente no sea del agrado de muchos creyentes, y cuya presencia, por otra parte, tampoco parece muy justificada en el contexto de esa obra. La carga ético-religiosa en obras posteriores sigue estando presente en sus 
ideas, incluso algunas veces da la impresión de ir por delante de los aspectos científicos ${ }^{2}$. Ese tipo de aproximaciones ha dado pie a que mucha gente intente proyectar la hipótesis Gaia hasta una dimensión que no entraba probablemente en las ideas iniciales de sus autores, lo cual obliga a Lovelock a afirmar que «De ningún modo veo a Gaia como un ser consciente, un Dios alternativo. Para mí Gaia está viva y es parte del universo inefable y yo soy una parte de ella.»

Lo antedicho no resta mérito al importante papel que la hipótesis Gaia ha tenido y tiene entre muchos científicos, como inspirador de una manera diferente de abordar el conocimiento. Desde ese punto de vista, Lynn Margulis en The Symbiotic Planet ${ }^{3}$, presenta un serio y prometedor enfoque científico que emana de Gaia. Sin embargo, en ese libro, no se hacen excesivas referencias a Gaia, sino que se utiliza una modalidad de pensar que podríamos denominar "Gaiana». Lo cual, teniendo en cuenta que ella fue en su momento cogeneradora de la hipótesis, da pie a pensar que sus ideas sobre la simbiosis fluyen de alguna manera un poco al margen de Gaia, que, socialmente hablando, probablemente ha cristalizado en demasía, y las dirige, con mayor firmeza, por los cauces científicos más ortodoxos.

Desde el punto de vista evolutivo, Lovelock nunca ha intentado crear una hipótesis que desbancara las ideas de Darwin: «Soy cien por cien Darwinista. Pero yo diría que Gaia es al Darwinismo lo que la relatividad es a la física de Newton. En ningún modo la reemplaza; meramente la extiende». A pesar de esa afirmación, muchos seguidores han encontrado en Gaia un nuevo combustible con el que atizar el fuego de la, casi eterna, discusión sobre la evolución.

Ante la consideración de una posible aceptación universal de la hipótesis Gaia, equiparable a la que tuvo la evolución Darwinista, existe una importante diferencia entre ambas. Las ideas de Darwin sonaban muy bien entre las elites de la Inglaterra del siglo XVIII y comienzos del XIX ${ }^{4}$. Dichas ideas daban soporte y justificación científica a lo que era el núcleo del discurso social de las clases dirigentes. Eso explica el increíble éxito editorial que tuvo «El origen de las especies» y que sorprendió al mismo Darwin. Ese discurso social subyace aún en buena medida en parte de la sociedad occidental actual, por lo cual el Darwinismo, en sus múltiples facetas, sigue teniendo un papel importante como idea rectora, porque sigue teniendo aplicación práctica en el contexto social.

Por el contrario, Gaia encontró el campo abonado en un sector social que estaba intentando en los años setenta romper con las estructuras sociales, tanto en Estados Unidos como en Europa. Los jóvenes de entonces se convirtieron después en adultos, impregnando sus profesiones con tintes de diversas ideologías. Hoy Gaia viaja con ellos en el tiempo y no pa- 
Algunas reflexiones sobre Gaia, evolución y cultura

rece que las nuevas generaciones comulguen con ella con el mismo fervor que las anteriores. Carente del soporte del discurso social imperante, es posible que tenga sus días contados. Claro que, como idea romántica, siempre puede volver a ser activada en cualquier momento.

El humano, especialmente si es de género masculino, suele tener tendencia a aclarar rápidamente las medias tintas y a tomar posición formando grupo, es decir, tribu, con los que considera más afines, distanciándose con ello de las demás tribus. Mediante ese procedimiento consigue aliviar las urgencias primitivas que nos llevan a excitarnos, incluso divertirnos, mediante la confrontación. De ese modo estamos mucho más dispuestos a tomar partido por el Lamarckismo, por el Darwinismo, o por la hipótesis Gaia, que a sumergirnos en la ardua, y nada divertida labor, de analizar lo que pueda haber de útil en cada una de las ideas. ¿Cómo explicar sino que pueda continuar un debate de más de dos siglos de duración, empleando unas armas intelectuales que casi no han variado, sobre todo si las comparamos con el avance ocurrido, durante ese mismo espacio de tiempo, en el campo tecnológico y en ciertos campos del conocimiento?

Probablemente es una osadía intentar conocer con precisión un tema, como el de la evolución, en el que estamos inexorablemente sumergidos y del que es imposible distanciarnos. Ni las dimensiones temporales que se manejan, ni las espaciales, ni la complejidad, están dentro de los rangos habituales del pensamiento ni de la acción. Es decir, tenemos frente a nosotros una tarea imposible, lo cual no quiere decir ni mucho menos que no merezca la pena intentarla. Precisamente ese tipo de retos exige tal grado de esfuerzo, que en sí mismo es ya un fruto. Por otra parte, ese mismo esfuerzo arrastra a campos de conocimiento vecinos que sin ese empuje permanecerían quizá aletargados.

No obstante, conviene no perder ni un solo momento la perspectiva de la enorme dificultad de reflexionar sobre la evolución de la biosfera. Se han escrito mucho millares de libros analizando el tema desde muchos puntos de vista, pero sin embargo, comparativamente, se ha prestado mucha menos atención a las circunstancias básicas que condicionan ese análisis. Se da más que por supuesto que el ser humano está dotado de la capacidad de abordarlo y, por lo tanto, la discusión se centra en las modalidades del análisis. Pero en estas páginas quisiera llamar la atención del lector sobre nuestras propias limitaciones, acudiendo a unos cuantos aspectos que son muy simples y bastante bien conocidos y que, sin embargo, se ignoran sistemáticamente en el discurso científico habitual.

En primer lugar plantear la obviedad de que la mente intelectual no dispone de una conexión directa con el exterior. Encerrada entre las pa- 
redes craneales, necesita de unos sensores que trasladen la información desde el exterior, un mundo extraño y agresivo, que no debe ponerse en contacto fisiológico directo con las neuronas. Dichos sensores tienen un rango limitado de actuación en función de las necesidades de la estructura corporal que los soporta y que tiene que trasladarlos de un sitio a otro, buscándose la vida entre otros seres que conforman el ecosistema. Por consiguiente, tienen que ser ligeros y proporcionar la información básica esencial, restringiendo al máximo su rango para evitar informaciones innecesarias, que ocupen lugar y distraigan de otras funciones. La actual explosión de actividad intelectual se hace detrayendo la atención de otras áreas y no es infrecuente que esa desatención repercuta en el equilibrio celular y conduzca a enfermedad. Por consiguiente debería tenerse en cuenta que, en alguna medida, estamos «forzando» nuestras capacidades naturales.

El desarrollo tecnológico ha conseguido extender extraordinariamente el rango de nuestros sensores y ha creado otros nuevos. Con ello hemos expandido brutalmente la cantidad de información a la que tenemos acceso, pero el problema real no es la cantidad de información sino la capacidad de procesamiento. Esa sigue estando limitada, pendiente de la aparición de un nuevo ser que esté un eslabón por encima nuestro en la evolución, por lo que nos vemos obligados a abordar los problemas de forma parcial: una limitación de primer orden al intentar comprender algo tan extraordinariamente complejo como la evolución.

Los procedimientos de comprensión tampoco suelen ser directos, sino que forman parte de una especie de «software» que se desarrolla en el individuo mediante el aprendizaje por ensayo y error, y por el condicionamiento del entorno social y de la cultura en el que se desenvuelva. Cada familia, cada grupo, cada cultura, desarrolla unos procedimientos propios que les son característicos y que utiliza constantemente para enjuiciar cualquier situación. Mediante esos procedimientos se crean en la mente de cada uno de nosotros modelos que imaginan cómo es el mundo que está fuera de nuestras paredes craneales.

Esos modelos son modelos «ad hoc», modelos creados en función del objeto o problema al que van dirigidos y en función de la intencionalidad del individuo. Fuera del contexto en el que fueron creados carecen probablemente de validez. El método científico surgió de la necesidad de una cierta estandarización de esos modelos. El franciscano Roger Bacon, en la Inglaterra del siglo XIII fue uno de los precursores en esa tarea. En el siglo XVI Francis Bacon estableció en esa misma línea las bases del actual método científico, pero, a diferencia de Roger, dejó al experimentador fuera del experimento, con lo cual se creó la ficción de la objetividad. 
Los protocolos de esa estandarización han resultado ser de una extraordinaria utilidad y sin duda han contribuido a impulsar la ciencia hasta los niveles actuales. Sin embargo, al igual que ocurre con nuestros sensores, su rango de utilidad está limitado por su propio diseño, algo que se hace evidente en cuanto se aborda un problema suficientemente complejo, como la evolución.

Por constitución, el ser humano está dotado de varios procedimientos para modelizar e intentar comprender la realidad exterior. Una parte del cerebro funciona en una modalidad que se podría llamar «secuencial» y que se aloja fundamentalmente en la parte izquierda del cerebro. Otra es de índole global, intuitiva, atemporal y reside aproximadamente en el hemisferio derecho, aunque las investigaciones recientes dibujan un panorama mucho más complejo. La llamada «lógica formal», que es la base estructurante del método científico, es un modelo, muy simple y sólido, que «corre» con mucha facilidad en el «hardware» del hemisferio izquierdo.

Sería de imaginar, que, ante un problema de una magnitud tan enorme como es el de la evolución, los seres humanos echásemos mano de todas las herramientas de que estamos dotados. Sin embargo, nos enfrentamos a él desde una óptica muy restringida, despreciando de entrada las capacidades del hemisferio derecho y dejando reducidas a un mínimo las del izquierdo. El encasquillamiento histórico en el que estamos metidos respecto al tema de la evolución, no podrá nunca desbloquearse mientras no estemos dispuestos a examinar con mente abierta todas las teorías, buscando aquellos aspectos que puedan ser de utilidad al paradigma de hoy, sean esos los que sean, sin miedo a romper el hilo del debate, y dejando a parte argumentaciones que pertenecen a un contexto social de otras épocas, y sabiendo que con ello estamos simplemente creando un nuevo modelo de utilidad limitada y con fecha de caducidad.

En ese nuevo enfoque habría que considerar algunos aspectos que vienen siendo sistemáticamente lanzados contra el modelo evolucionista, por así decir «occidental», por los modelos creados por otros grupos socioculturales. Entre ellos destaca el «diseño». Es constante encontrar en la naturaleza artilujios que participan del espíritu del «ingenio» humano; por ejemplo, el lóbulo membranoso que tiene el rape (Lophius piscatorius) en la extremidad de una de las varias espinas que salen de su cabeza y que utiliza como señuelo para capturar sus presas. Es decir, el mismo procedimiento que empleamos los humanos para pescar con señuelo. Nadie duda, al ver una caña de pescar y un aparejo, que eso es fruto de un diseño intencionado. Sin embargo, sistemáticamente nos cerramos en banda a la posibilidad de que su equivalente en el pez pueda responder a una intencionalidad. Esa actitud es fruto del «modelo» que 
estamos empleando y que incluye aspectos religioso-culturales anclados en el pasado. Ejemplos como ese hay miles y hay gente que se dedica a arrojarlos al mundo escrito para intentar desprestigiar la teoría de la evolución ${ }^{5}$. Aunque es muy interesante tratar de ver con ojos diferentes ciertos hechos, generalmente nos encontramos, de nuevo, ante modelos que pretenden, más que clarificar el proceso de comprensión, sustituir unos modelos absolutistas por otros. En mi opinión, estamos sobrados de ese tipo de procedimientos y lo que necesitamos ahora es, fundamentalmente, mucha más humildad, flexibilidad, espíritu crítico y abrir los procedimientos para incorporar otras herramientas que están siendo empleadas en otros campos científicos, como la teoría de la complejidad ${ }^{6}$.

Jalaludin Rumi, sabio y místico del siglo XIII que vivía en la ciudad de Konia, escribió lo siguiente: «En el origen fuiste arcilla. Partiendo de ser un mineral, te hiciste vegetal. De vegetal te hiciste animal, y de animal, hombre. Durante estos periodos el hombre no sabía a dónde iba, sin embargo estaba siendo transportado en un largo viaje. Y todavía tendrás que pasar por cien mundos diferentes.» Visto así parece una idea precursora de las teorías sobre la evolución, pero no hay que perder de vista el uso habitual que se hacía en aquella época de la analogía, por lo que no podemos estar seguros que necesariamente se estuviese refiriendo a lo que nos referiríamos ahora con esa fraseología. Es decir, su interpretación requiere conocer previamente las claves culturales y específicas del grupo para el que fueron escritas, conocer su modelo. Todo lo antedicho en estas páginas queda resumido, y al mismo tiempo ampliado, en su conocida historia del elefante y los ciegos, en el que tras examinar sus distintas partes, cada uno se empeña en hacer valer su modelo e imponerlo a los demás, afirmando que aquella entidad era un conjunto de rollos de alfombra, o unos tapices, o unos pilares, o cuerdas, según qué parte del animal hubiese explorado. En este caso, Lamarck, Darwin, Lovelock y todos nosotros, seríamos esos ciegos, y la biosfera y su evolución, el elefante.

\section{Bibliografía}

1 Lovelock, J. 1993: Las Edades de Gaia. Tusquets Editores, Barcelona.

2 A way of life for agnostics, Gaia Circular, Summer 1999. Dept. of Environmental Sciences, University of East London.

3 Margulis, L. 1998: The Symbiotic Planet. Phoenix, Orion Books, Londres.

4 SAndín, M. 2000: Sobre una redundancia: el Darwinismo social. Asclepio, V LII-2.

5 Por ejemplo, véase YAHYA, H. 2000.The Miracle in the Ant. The series of facts demolishing the lie of evolution-1. Ta-Ha Publishers, Londres.

${ }^{6}$ Kauffman, S. 1993. The Origins of Order: self-organization and selection in evolution. Oxford University Press. 